\title{
Propiedades probióticas de Lactobacillus spp aislados de biopsias gástricas de pacientes con y sin infección por Helicobacter pylori
}

\author{
Apolinaria García C ${ }^{1 a}$, Patricio Henríquez $A^{1 a}$, \\ Carolina Retamal $\mathbf{R}^{\mathbf{l b}}$, Susana Pineda $C^{\mathbf{l a}}$, \\ Carolina Delgado Sch², Carlos González C ${ }^{1 a}$.
}

Probiotic properties of Lactobacillus spp isolated from gastric biopsies of Helicobacter pylori infected and non-infected individuals

Background: Gastric microbiota is a complex ecosystem, composed by diverse microorganisms, where H pylori and Lactobacillus are relevant species. Aim: To study the probiotic properties of Lactobacillus spp isolated from gastric biopsies of subjects bearing or not $H$ pylori. Materials and Methods: Gastric biopsies from 197 individuals that consulted for gastrointestinal disorders were cultivated in media specific for H pylori and Lactobacillus spp. Probiotic properties of lactobacilli isolates and histological diagnosis of H pylori colonized patients were performed. Results: Among 197 individuals evaluated, H pylori was detected in 43\%, Lactobacillus spp in 24\%, while $8 \%$ presented both bacteria simultaneously. Forty one percent of the Lactobacillus spp strains produced bydrogen peroxide, 83\% presented a highly bydrophobic surface and $32 \%$ had an inbibitory effect upon H pylori ATCC 43504, even greater than Lactobacillus LGG or LAI reference strains. The main pathological diagnosis of patients was chronic non-atrophic gastritis (73\%) followed by intestinal metaplasia (19\%). A similar proportion of both conditions were observed in patients colonized by Lactobacillus spp, while not colonized individuals developed preferentially chronic non-atrophic gastritis $(p=0.002)$. Conclusions: Lactobacillus spp and $H$ pylori were mutually excluded, suggesting a competitive colonization model between probiotic bacteria and pathogens (Rev Méd Chile 2009; 137: 369-76).

(Key words: Helicobacter pylori; Lactobacillus; Probiotics)

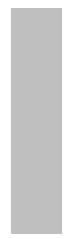

\footnotetext{
Recibido el 12 de mayo, 2008. Aceptado el 27 de noviembre, 2008.

Este trabajo fue financiado por el Proyecto Fondef DO3i1105.

${ }^{1}$ Laboratorio de Patogenicidad Bacteriana, Departamento de Microbiología, Facultad de Ciencias Biológicas. Universidad de Concepción. ${ }^{2}$ Departamento de Especialidades Médicas, Facultad de Medicina, Universidad de Concepción. Concepción, Chile.

${ }^{a}$ Bioquímica, Doctora $(\mathrm{PhD})$ en Ciencias Biológicas

${ }^{\mathrm{b}}$ Tecnólogo Médico
}

Correspondencia a: Dra. Apolinaria García. Departamento de Microbiología, Facultad de Ciencias Biológicas, Universidad de Concepción. Casilla 160-C, Concepción, Chile. Teléfono: 56

(41) 2204144. Fax: 56 (41) 2245975. E mail: apgarcia@udec.cl 
$\mathrm{L}$ a microbiota gastrointestinal es un complejo ecosistema compuesto por microorganismos capaces de promover efectos beneficiosos para la salud y otros, considerados patógenos por su capacidad de afectar al hospedero ${ }^{1}$. Entre sus funciones destacan: 1) fermentación de residuos de la dieta y mucinas endógenas; 2) recuperación de energía por generación de ácidos grasos de cadena corta; 3) protección contra colonización e invasión de patógenos y 4) desarrollo, estimulación y modulación del sistema inmune ${ }^{1,2}$.

Parte de esta microbiota gastrointestinal es la microbiota gástrica, que se ha caracterizado en base al cultivo de biopsias gástricas o jugos gástricos, identificándose especies de los phyla Firmicutes, Proteobacteria, Actynobacteria y Fusobacteria y, levaduras ${ }^{3,4}$. Molecularmente se ha encontrado una comunidad de 128 filotipos, donde 10\% nunca había sido descrito para esta región ${ }^{5-9}$. Entre las especies más caracterizadas están Helicobacter pylori, Lactobacillus spp, Bifidobacterium spp, Peptostreptococcus spp, Sarcina spp, especies no patógenas de Streptococcus spp y Staphylococcus spp, y levaduras como Candida spp. ${ }^{6-9}$.

H pylori es la especie más estudiada por asociarse con patologías gástricas como úlcera péptica, linfoma de MALT y cáncer gástrico ${ }^{10-12}$. Este microorganismo se encuentra entre 70\% y $90 \%$ en población de países en vías de desarrollo y en $25 \%$ a $50 \%$ en países desarrollados, produciendo mayoritariamente infección asintomática ${ }^{13}$.

Otro género que ha concitado interés es Lactobacillus, pues está constituido por bacterias acidolácticas, que crecen en diversidad de condiciones y forman parte de la microbiota normal de la boca, tracto gastrointestinal y genitourinario humano $5,14,15$. En el estómago es posible encontrarlo en concentraciones de 0 a $10^{3}$ UFC por $\mathrm{ml}$ de contenido gástrico ${ }^{16}$. Sus características ácido-resistentes, le ayudan a persistir en el estómago más tiempo que otras bacterias ${ }^{17}$. Lactobacillus spp se utiliza como bacteria probiótica, término que se refiere a microorganismos no patogénicos que, ingeridos en cantidad adecuada, proporcionan características de la microbiota normal del hospedero ${ }^{1}$. Este microorganismo puede ayudar a prevenir o tratar diversas enfermedades gastrointestinales, ya sea, impidiendo la ocupación de sitios específicos, produciendo sustancias antagónicas o modulando la respuesta inmune ${ }^{18-21}$. Producen metabolitos conocidos como sustancias antagónicas, entre ellos, ácidos grasos de cadena corta (AGCCs) y peróxido de hidrógeno $\left(\mathrm{H}_{2} \mathrm{O}_{2}\right)^{22,23}$. Uno de los AGCCs es el ácido láctico, que disminuye el pH impidiendo el desarrollo de diversos microorganismos. Su capacidad para colonizar epitelios también ha sido destacada y se relaciona con una alta hidrofobicidad de superficie, como se ha descrito para otros microorganismos con propiedades similares ${ }^{15,24}$.

Una completa revisión de Gotteland y cols (2006), resume la evidencia experimental que demuestra las propiedades anti- $H$ pylori que presentan cepas específicas de Lactobacillus ${ }^{25}$. Entre éstas, señala que Lactobacillus spp puede ejercer efecto bactericida sobre $H$ pylori a través de la liberación de bacteriocinas e inhibir su adherencia a células epiteliales. Se ha demostrado que también presenta efecto anti $H$ pylori inhibiendo su ureasa $22,26,27$ o produciendo $\mathrm{H}_{2} \mathrm{O}_{2}$, molécula que induce muerte bacteriana mediante un mecanismo de defensa antimicrobiano inespecífico ${ }^{15,23}$.

La prevalencia de Lactobacillus en el estómago está poco documentada y, no se dispone de información sobre propiedades probióticas de cepas aisladas desde el estómago humano. Tampoco se ha informado en literatura la posibilidad de coexistencia o no de H pylori y Lactobacillus spp en el epitelio gástrico humano.

El objetivo de este trabajo fue determinar algunas propiedades probióticas de Lactobacillus spp aislados desde biopsias gástricas de individuos infectados y no infectados por $H$ pylori, y establecer si existe antagonismo entre ambas especies en este hábitat.

\section{MATERIAL Y MÉTODO}

Población en estudio. Se incluyeron 197 pacientes chilenos mayores de 15 años (44,5 $\pm 21,04)$, con indicación de endoscopia digestiva alta, a los cuales se les tomaron dos biopsias gástricas, previo consentimiento informado, las cuales se utilizaron para estudio microbiológico e histopatológico. Las biopsias fueron transportadas en suero fisiológico estéril hasta el Laboratorio de Patogenicidad Bacteriana (Universidad de Concepción) donde fueron procesadas. Se siguieron 
las recomendaciones del European Helicobacter pylori Study Group (1997)28. Este estudio fue aprobado por el Comité de Ética de la Facultad de Medicina, Universidad de Concepción.

Condiciones de cultivo e identificación de cepas bacterianas. Las biopsias mantenidas a $4^{\circ} \mathrm{C}$ en "suero fisiológico" fueron maceradas y homogeneizadas en mortero. Para el aislamiento de $H$ pylori $0,1 \mathrm{ml}$ del homogeneizado fue sembrado en agar Columbia (Oxoid) suplementado con 5\% de sangre de caballo y DENT (Oxoid), incubando a $37^{\circ} \mathrm{C}$ en microaerofilia (CampyGen, Oxoid) durante 5 dias $^{29}$. Las colonias se identificaron mediante prueba de ureasa, catalasa y tinción de Gram. Se consideraron pacientes positivos a la infección por $H$ pylori los que presentaron cultivo positivo.

Para el aislamiento de Lactobacillus spp se inocularon $0,1 \mathrm{ml}$ del homogeneizado en $1 \mathrm{ml}$ de caldo MRS (Oxoid) y se incubaron a $37^{\circ} \mathrm{C}$ en atmósfera con $10 \%$ de $\mathrm{CO}_{2}$ durante 2 días $^{30}$. Los caldos que resultaron con crecimiento positivo fueron sembrados en placas de agar MRS e incubados en las condiciones ya descritas. A las colonias sospechosas de Lactobacillus spp se les realizó tinción de Gram, prueba de catalasa y oxidasa $^{30}$. Los pacientes colonizados por Lactobacillus spp fueron aquéllos que presentaron cultivo en agar MRS positivo.

Las cepas de $H$ pylori fueron almacenadas en caldo tioglicolato (MERCK) y las de lactobacilos en caldo MRS, con $25 \%$ de glicerol a $-80^{\circ} \mathrm{C}$ para estudios posteriores ${ }^{31}$. Para realizar los ensayos de propiedades probióticas, las cepas de Lactobacillus spp se reactivaron mediante 2 subcultivos consecutivos de 1 día cada uno, en caldo MRS a $37^{\circ} \mathrm{C}$.

Como cepa control de $H$ pylori se utilizó la cepa ATCC 43504 y para Lactobacillus se utilizaron dos cepas de referencia: Lactobacillus cepa GG (LGG) y Lactobacillus johnsonii LA1 (LA1).

Estudio histopatológico. Los cortes histológicos de antro y cuerpo del estómago fueron teñidos con hematoxilina-eosina y giemsa modificado, de acuerdo a pautas previamente publicadas ${ }^{32}$. Dicho examen fue realizado por 2 observadores en forma independiente, aceptándose sólo casos con todo el espesor de la mucosa. Se realizó el diagnóstico final de acuerdo a la clasificación de Sydney ${ }^{32}$.

Producción de peróxido de hidrógeno. Se utilizó el protocolo descrito por Felten y $\mathrm{col}^{33}$. Las cepas de Lactobacillus spp fueron sembradas sobre placas con $20 \mathrm{ml}$ de agar MRS, suplementadas con $5 \mathrm{mg}$ de 3,3',4,4'-tetrametilbenzidina (Sigma) y 0,2 mg de peroxidase horse radish (Sigma). La identificación de bacterias productoras de $\mathrm{H}_{2} \mathrm{O}_{2}$ se realizó después de la incubación, realizada en las condiciones descritas anteriormente, considerando dos categorías: no productora y productora de $\mathrm{H}_{2} \mathrm{O}_{2}$.

Hidrofobicidad de superficie bacteriana. Se utilizó el ensayo de partición en solventes orgánicos MATH (Microbial Adbesion to Hydrocarbons) ${ }^{34}$. A $1,2 \mathrm{ml}$ de suspensión bacteriana, de absorbancia conocida $\left(\mathrm{DO}_{600}=0,4-0,7\right)$, se adicionó $0,3 \mathrm{ml}$ de Xileno y se mezclaron con vórtex durante $1,5 \mathrm{~min}$. Se dejó reposar durante $15 \mathrm{~min}$, se removió la fase acuosa y se midió la absorbancia a $600 \mathrm{~nm}$.

El porcentaje de hidrofobicidad $(\% \mathrm{H})$ fue calculado según ecuación de Ocaña y $\mathrm{col}^{15}$. Los valores obtenidos permiten clasificar a las cepas con una hidrofobicidad alta (71\%-100\%); media $(36 \%-70 \%)$ y baja $(0 \%-35 \%)^{15}$.

Inbibición del crecimiento de H pylori mediada por cepas de Lactobacillus spp. Este ensayo fue realizado de forma similar a lo descrito previamente ${ }^{22}$. En placas de agar Müeller-Hinton, suplementadas con $5 \%$ de sangre de caballo desfibrinada, se realizaron orificios de $5 \mathrm{~mm}$ de diámetro. Posteriormente, se sembró $H$ pylori ATCC 43504 en tapiz y en los orificios se inocularon $0,03 \mathrm{ml}$ de Lactobacillus spp reactivados como se describió anteriormente. Las placas fueron incubadas en las condiciones ya descritas para H pylori. Finalmente, se midieron los halos de inhibición, los que fueron comparados con los halos producidos por las cepas de referencia utilizadas.

Estadística. Los resultados se recolectaron en tablas de frecuencia y tablas de contingencia y fueron analizados según test de Chi cuadrado y correlaciones, con intervalos de confianza de 95\%. El análisis se completó usando software estadístico Statistical Package for Social Sciences (SPSS versión 10.0). 


\section{Resultados}

Aislamiento de Lactobacillus spp y Helicobacter pylori desde biopsias gástricas. Del total de 197 pacientes, 42,6\% (84 pacientes) estaba infectado por $H$ pylori (Figura 1A). Los pacientes colonizados por Lactobacillus spp correspondieron a 24,4\%, que equivale a 48 pacientes (Figura 1B).

El análisis estadístico mostró que la presencia de ambas bacterias no presenta una relación significativa (P: 0,067); sin embargo, al realizar una correlación entre ambas variables se observó que existe una relación negativa, pero débil $(-0,131)$. Esto quiere decir que, en los pacientes $H$ pylori positivos, Lactobacillus spp se encontró en bajo porcentaje (Figura 2A) y en aquellos que presentaban Lactobacillus spp, hubo bajo porcentaje de $H$ pylori (Figura 2B).

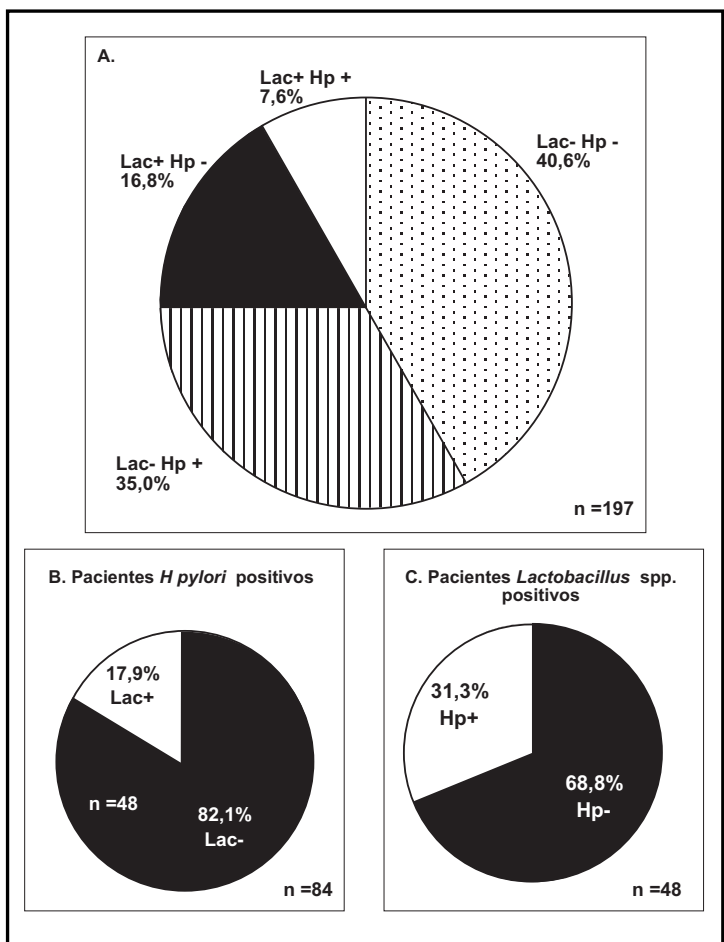

Figura 1. Prevalencia de Lactobacillus spp. y H pylori en individuos sintomáticos. (A) Distribución por grupos (\%) según microorganismo presente; (B) Porcentaje de individuos con o sin Lactobacillus spp. en pacientes infectados por H pylori; (C) Porcentaje de individuos con o sin infección por $H$ pylori, colonizados por Lactobacillus spp.
Caracterización de propiedades probióticas de Lactobacillus spp aislados desde biopsias gástricas Producción de peróxido de bidrógeno: Las cepas de Lactobacillus spp presentaron una variada producción de $\mathrm{H}_{2} \mathrm{O}_{2}$ (datos no mostrados). De las 48 cepas aisladas, $41,7 \%$ (20 cepas) produjo $\mathrm{H}_{2} \mathrm{O}_{2}$. Un bajo porcentaje de los pacientes de los cuales se aislaron las cepas productoras de $\mathrm{H}_{2} \mathrm{O}_{2}$ estaba infectado por H pylori (Figura 3). Los pacientes colonizados con cepas no productoras de $\mathrm{H}_{2} \mathrm{O}_{2}$ también mostraron una baja prevalencia de infección por $H$ pylori. Sin embargo, la proporción de individuos infectados con Hpylori en este último grupo, fue mayor que en los pacientes que presentan cepas de Lactobacillus productoras de $\mathrm{H}_{2} \mathrm{O}_{2}$. Estas diferencias no fueron significativas (P: 0,301). En las cepas control de Lactobacillus no se detectó producción de $\mathrm{H}_{2} \mathrm{O}_{2}$.

Hidrofobicidad de superficie bacteriana: 83,3\% (40/48) de los Lactobacillus spp presentó alta hidrofobicidad de superficie, 8,3\% presentó hidrofobicidad de superficie media e igual porcentaje mostró una baja hidrofobicidad. Las cepas controles mostraron alta hidrofobicidad de superficie bacteriana (datos no mostrados).

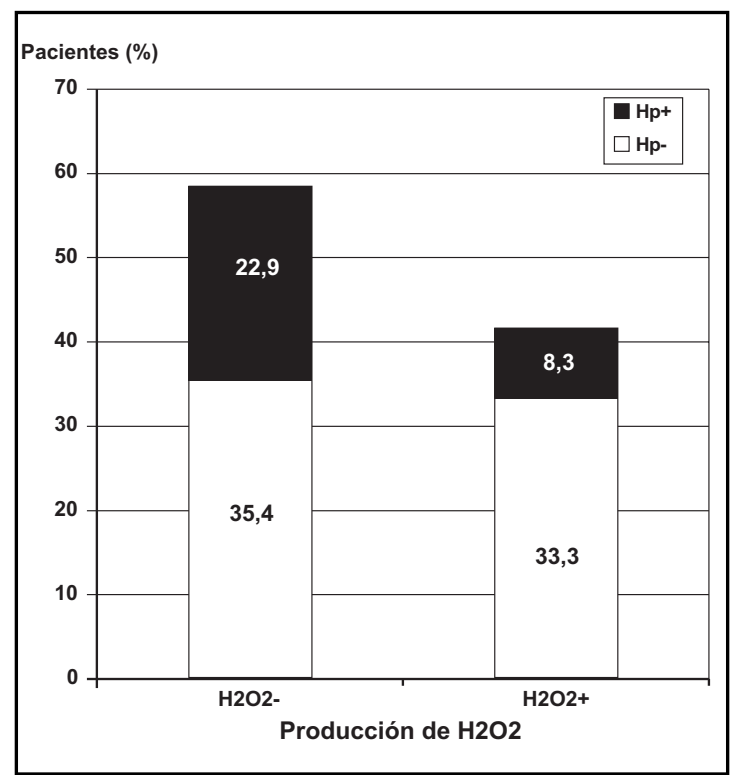

Figura 2. Distribución de $H$ pylori en pacientes que presentaban Lactobacillus spp. productores $\left(\mathrm{H}_{2} \mathrm{O}_{2}+\right)$ y no productores de peróxido de hidrógeno $\left(\mathrm{H}_{2} \mathrm{O}_{2}-\right)$ (Hp: H pylori). 


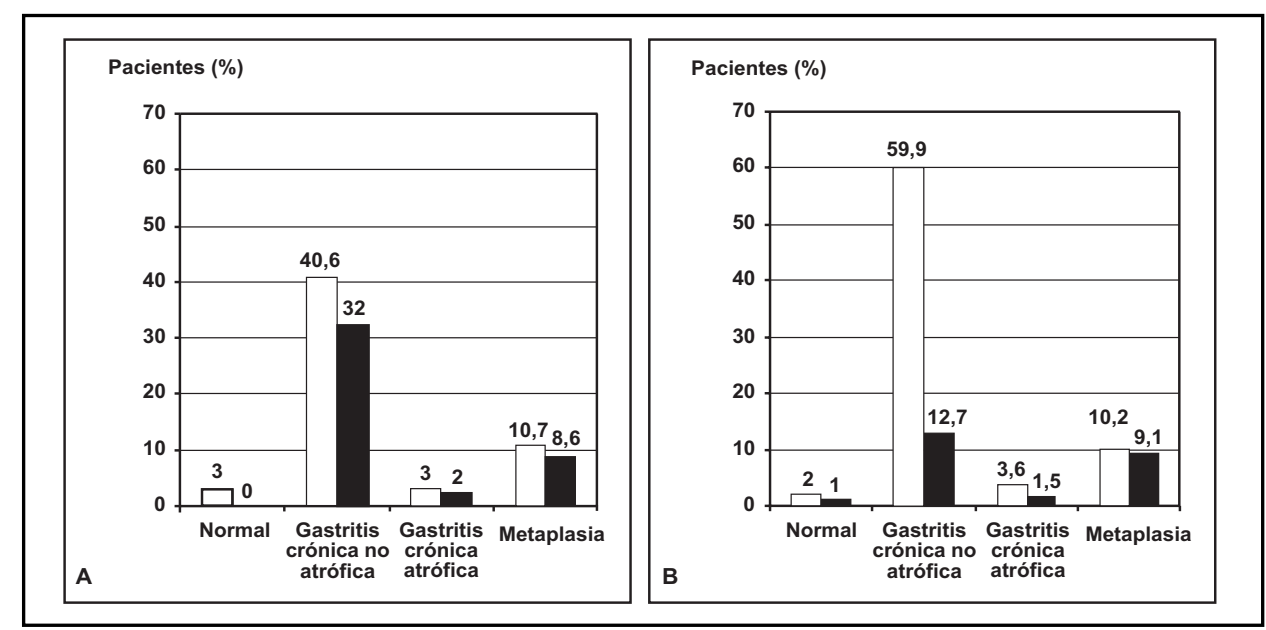

Figura 3. Distribución de los diagnósticos histopatológicos en el grupo de pacientes H pylori positivos (A) y Lactobacillus spp. positivos (B). Las barras blancas indican ausencia de bacterias y las barras negras, presencia de ellas.

Inbibición del crecimiento de H pylori mediada por Lactobacillus spp: De 38 cepas de Lactobacillus spp analizadas, $12(31,6 \%)$ presentaron efecto inhibitorio sobre $H$ pylori ATCC 43504, superior al de las cepas control utilizadas (LGG $2.0 \mathrm{~cm}$ y LA1 $2,4 \mathrm{~cm})$. Veintidós de las cepas ensayadas $(57,9 \%)$ presentaron halos de inhibición superior a 1,0 cm de diámetro (Tabla 1).

Diagnóstico bistopatológico y su asociación a $H$ pylori y Lactobacillus spp. La clasificación de los parámetros histopatológicos de las biopsias gástricas analizadas arrojó 4 grupos, por orden creciente de severidad fueron: normal, gastritis crónica no atrófica, gastritis crónica atrófica y metaplasia intestinal. El diagnóstico histopatológico más frecuente fue gastritis crónica no atrófica, con $72,6 \%$ (143), seguido de metaplasma intestinal, con 19,3\% (38) (Figura 4). De todas las biopsias analizadas sólo 3,0\% $\%^{6}$ estaba dentro de parámetros histológicos normales, siendo además, negativas para H pylori (Figura 4).

La mayoría de los pacientes infectados por $H$ pylori presentaba gastritis crónica no atrófica, pero la distribución de los diagnósticos histopatológicos fue similar para pacientes infectados y no infectados, con diferencias no significativas ( $\mathrm{P}$ : 0,197) (Figura 1A). En el caso de Lactobacillus spp se encontró una diferencia significativa ( $P$ : 0,002) en la distribución de los diagnósticos histopatológicos con respecto a la presencia o no de la bacteria. Así, la mayoría de los pacientes colonizados por esta bacteria presentaron similar porcentaje de gastritis crónica no atrófica y metaplasia intestinal (Figura 1B). En cambio, en el caso de los pacientes no colonizados con Lactobacillus spp se observó que la mayoría de los pacientes presentaban gastritis crónica no atrófica (Figura 1B).

Tabla 1. H alos de inhibición producidos por cepas de Lactobacillus spp., aislados desde biopsias gástricas

\begin{tabular}{|lcccc|}
\hline & & \multicolumn{2}{c|}{ Halo de Inhibición $(\mathbf{c m})$} & $\mathbf{2}$ ) \\
& $\mathbf{0 - 1 , 0}$ & $\mathbf{1 , 1 - 2 , 0}$ & $\mathbf{2 , 1 - 3 , 0}$ & $\mathbf{3}, \mathbf{0}$ \\
\hline Lactobacillus spp. (número) & 16 & 10 & 9 & 3 \\
Lactobacillus spp. (\%) & 42,1 & 26,3 & 23,7 & 7,9 \\
\hline
\end{tabular}




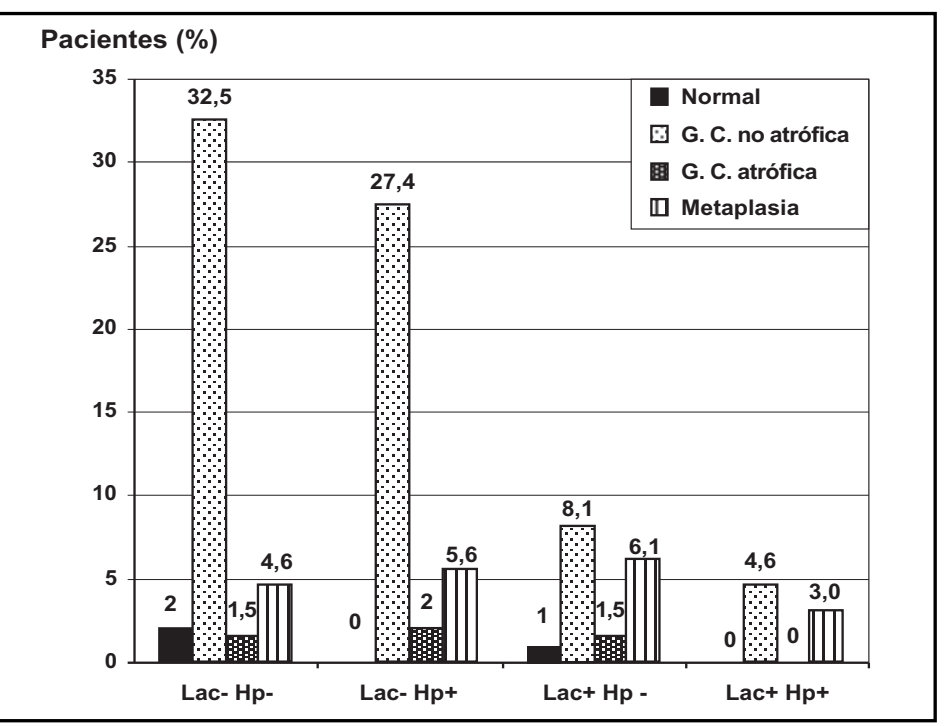

Figura 4. Distribución de los diagnósticos histopatológicos en relación a la ausencia o presencia de $H$ pylori y Lactobacillus spp. (Lac: Lactobacillus spp; Hp: H pylori; G.C.: gastritis crónica).

Al analizar los diagnósticos histopatológicos en relación a la presencia o ausencia de ambos microorganismos, se observó que cuando ambas especies estaban ausentes el diagnóstico más recurrente fue gastritis crónica no atrófica, al igual que en el grupo de pacientes que presentaban sólo H pylori (Figura 4). No obstante, cuando los pacientes fueron colonizados solamente por Lactobacillus spp, se observaron porcentajes similares de gastritis crónica no atrófica y metaplasia intestinal (Figura 4). Al estar ambas especies presentes, los diagnósticos eran gastritis crónica no atrófica, en mayor porcentaje, y metaplasia intestinal, correspondiendo este grupo a un bajo porcentaje de pacientes, 7,6\% 15 (Figura 4).

\section{DisCUSIÓN}

Si bien existe una gran cantidad de estudios respecto de los Lactobacillus spp como agentes probióticos $^{1,2,20}$, no encontramos uno que señale la prevalencia de esta bacteria en la mucosa gástrica, ni el mecanismo de colonización de la misma que aún es incierto ${ }^{25}$. Por ello, podemos indicar que nuestros resultados arrojan un porcentaje estimativo de la presencia de este género bacteriano en la región gástrica, sirviendo como referencia para estudios posteriores.

En cuanto a la presencia de H pylori, encontramos diferencias con resultados publicados por otros autores, donde se señala que en países en vías de desarrollo la prevalencia de este microorganismo se encuentra entre 75\% y $90 \% 35,36$. En nuestro trabajo fue menor, debido a que la detección se basó en el cultivo de $H$ pylori y no en métodos inmunológicos, los que detectan formas viables o no viables de la bacteria y que no indica si la infección es actual o pasada.

En la literatura se describen distintas propiedades probióticas de los Lactobacillus spp. Nosotros determinamos hidrofobicidad de superficie, para evaluar la capacidad de Lactobacillus spp para interactuar con superficies epiteliales, encontrando que la mayoría de las cepas aisladas presentaban alta hidrofobicidad, concordante con las características que deben presentar bacterias que colonizan superficies epiteliales ${ }^{15,24}$.

También determinamos la producción de $\mathrm{H}_{2} \mathrm{O}_{2}$ para evaluar la síntesis de una sustancia antimicrobiana, encontrando un bajo porcentaje en relación a Lactobacillus spp aislados del tracto vaginal de mujeres sanas, donde se reporta $86 \%$ 
de cepas productoras de $\mathrm{H}_{2} \mathrm{O}_{2}{ }^{31}$. Sin embargo, al analizar sólo el grupo de Lactobacillus spp productores de este compuesto, podemos observar que existe una menor presencia de $H$ pylori, a diferencia del grupo que no produce $\mathrm{H}_{2} \mathrm{O}_{2}$, lo que sugiere que la producción de este antimicrobiano estaría jugando un rol en la inhibición del crecimiento de $H$ pylori en el estómago.

Otro de los aspectos importantes de este trabajo fue analizar la coexistencia de Lactobacillus spp y de H pylori en los pacientes estudiados. Si bien el test de chi cuadrado señala que la relación de coexistencia o no de ambas bacterias no es significativa (P: 0,067), la correlación de Pearson indica que existe una tendencia a no coexistir simultáneamente ambas en la región gástrica $(-0,131)$.

Si analizamos los diagnósticos histopatológicos, podemos señalar que el diagnóstico más recurrente fue gastritis crónica no atrófica, el menos severo según la escala establecida. Sin embargo, el segundo lugar en frecuencia fue metaplasia intestinal, el que presenta mayor presencia de Lactobacillus spp, lo que sugiere que esta bacteria se establece

\section{REFERENCIAS}

1. Kolida S, Saulnier DM, Gibson GR. Gastrointestinal microflora: probiotics. Adv Appl Microbiol 2006; 59: 187-219.

2. Quera R, Quigley E, Madrid A. El rol de los prebióticos, probióticos y simbióticos en gastroenterología. Gastr Latinoam 2005; 16: 218-28.

3. Adamsson I, Nord CE, Lunquist P, Lundquist P, Sjostedt S, EdLund C. Comparative effects of omeprazole, amoxycillin plus metronidazole versus omeprazole, clarithromycin plus metronidazole on the oral, gastric and intestinal microflora in Helicobacter pylori infected patients. J Antimicrob Chemother 1999; 44: 629-40.

4. SaVage DC. Microbial ecology of the gastrointestinal tract. Annu Rev Microbiol 1977; 31: 107-33.

5. Biк EM, Eckburg PB, Gill SR, Nelson KE, Purdom EA, Francois F ET AL. Molecular analysis of the bacterial microbiota in the human stomach. Proc Natl Acad Sci USA 2006; 103: 732-7.

6. Zoetendal EG, Collier CT, Koike S, Mackie Ri, Gaskins HR. Molecular Ecological Analysis of the Gastrointestinal Microbiota: A Review. J Nutr 2004; 134: 465-72.

7. Hugenholz P, Goebel BM, PACE NR. Impact of cultureindependent studies on the emerging phylogenetic en la mucosa gástrica, cuando ésta se encuentra lesionada. Este interesante hallazgo se correlaciona con lo encontrado por otros investigadores, quienes observaron que estas especies están presentes en biopsias gástricas de pacientes que presentan patologías gástricas como erosiones, úlceras, linfomas y adenocarcinoma ${ }^{37}$. El hecho que especies consideradas como microorganismos probióticos se encuentren en estados patológicos neoplásicos o de alteración tisular, podría explicarse debido a que los cambios como aumento del pH y exposición de la superficie celular, favorecen el crecimiento de estas bacterias. Además, estudios han demostrado el papel que juegan estas bacterias en procesos de regeneración tisular ${ }^{16}$.

El aislamiento y caracterización de cepas de Lactobacillus spp y de $H$ pylori del estómago humano contribuirá a avanzar en el conocimiento de la interacción de estas bacterias en el hombre. Los estudios actuales que analizan la colonización del estómago por lactobacilos utilizan alimentos probióticos que contienen cepas de origen intestinal, cuyo mecanismo de protección frente a la colonización por $H$ pylori es desconocido ${ }^{38-40}$.

view of bacterial diversity. J Bacteriol 1998; 180 : 4765-74

8. PACE NR. A molecular view of microbial diversity and the biosphere. Science 1997; 276: 734-40.

9. Amann RI, Ludwig W, Schleifer KH. Phylogenetic identification and in situ detection of individual microbial cells without cultivation. Microbiol Rev 1995; 59: 143-69.

10. Blaser MJ, Atherton J. Helicobacter pylori persistence: biology and disease. J Clin Invest 2004; 113: 32133.

11. Suerbaum S, Michetti P. Helicobacter pylori infection. N Engl J Med 2002; 347: 1175-86.

12. Marshall BJ, WarRen JR. Unidentified curved bacilli in the stomach of patients with gastritis and peptic ulceration. Lancet 1984; 1: 1311-4.

13. Hamilton-Miller JMT. The role the probiotics in the tratament and prevention of Helicobacter pylori infection. Int J Antimicrob Angents 2002; 22: 360-6.

14. Jernberg C, Sullivan A, Edlund C, Jansson J. Monitoring of antibiotic-induced alterations in the human intestinal microflora and detection of probiótica strains by use of terminal restriction fragment length polymorphism. Appl Environ Microbiol 2005; 71 : 501-6.

15. Ocaña VS, Bru E, De Rui Holgado AP, Nader-Macias ME. Surface characteristics of lactobacilli isolated 
from human vagina. J Gen Appl Microbiol 1999; 45 : 203-12.

16. Kabir AM, Aiba Y, Takagi A, Kamiya S, Miwa T, Koga Y. Prevention of Helicobacter pylori infection by lactobacilli in a gnotobiotic murine model. Gut 1997; 41: 49-55.

17. Marteau P, Minekus M, Havenaar R, Huis in't Veld JH. Survival of lactic acid bacteria in a dynamic model of the stomach and small intestine: validation and the effects of bile. J Dairy Sci 1997; 80: 1031-7.

18. Garrido D, Suau A, Pochart P, Crochet S, Gotteland M. Modulation of the fecal microbiota by the intake of a Lactobacillus jobnsonii La1-containing product in human volunteers. FEMS Microbiol Lett 2005; 248: 249-56.

19. SARTOR RB. Probiotic therapy of intestinal inflammation and infections. Curr Opin Gastroenterol 2005; 21: $44-50$

20. Sullivan A, Nord C. Probiotics and gastrointestinal diseases. I Inter Med 2005; 257: 78-92.

21. Servin AL, Coconnier MH. Adhesion of probiotic strains to the intestinal mucosa and interaction with pathogens. Best Pract Res Clin Gastroenterol 2003; 17: $741-54$

22. Sgouras D, Maragkoudakis P, Petraki K. In vitro and in vivo inhibition of Helicobacter pylori by Lactobacillus casei strain Shirota. Appl Environ Microbiol 2004; 70: 518-26.

23. Piard JC, Desmazeaud M. Inhibiting factors produced by lactic acid bacteria. 1. Oxygen metabolites and catabolism end-products. Le Lait 1991; 71: 525-41.

24. Rosemberg M, Judes H, Weiss E. Cell surface hydrophobicity of dental plaque microrganisms in situ. Infect Immun 1983; 42: 831-4.

25. Gotteland M, Brunser O, Cruchet S. Systematic review: are probiotics useful in controlling gastric colonization by Helicobacter pylori? Aliment Pharmacol Ther 2006; 23: 1077-86.

26. Aiba Y, Suzuki N, Kabir AM, Takagi A, Koga Y. Lactic acid-mediated supresión of Helicobacter pylori by the oral administration of Lactobacillus salivarius as a probiotic in a gnotobiotic model. Am J Gastroenterol 1998; 93: 2097-101.

27. Bathia SJ, Kochar N, Abraham P, Fair NG, Mehta AP. Lactobacillus acidophilus inhibits growth of Campylobacter pylori in vitro. J Clin Microbiol 1989; 27 : 2328-30.
28. Working Party of the European Helicobacter Pylori Study Group. Guidelines for clinical trials in Helicobacter pylori infection. Gut 1997; 41: S3.

29. Gilbert J, Ramakrishna J, Sunderman FW, Wright A, Plut A. Protein Hpn: Cloning and characterization of a histidine-rich metal-binding polypeptide in Helicobacter pylori and Helicobacter mustelae. Infect Immun 1995; 63: 2682-8.

30. SHARPE E. The Genus Lactobacillus. En: The Prokaryotes. A handbook on habitats, isolation, and identification of bacteria, Volumen II, Primera edición. Starr M, Stolp H, Trüper H, Balows A, Schlegel H. (Eds.). Springer-Verlag Berlin Heidelberg. 1981; 1653-79.

31. Angeles-López M, García-Cano E, Aquino C. Hydrogen peroxide production and resistance to nonoxinol-9 in Lactobacillus spp. Isolated from the vagina of reproductive age women. Rev Latinoam Microbiol 2001; 43: 171-6.

32. Dixon MF, Genta RM, Yardley JH, Correa P. Classification and grading of gastritis. The updated Sydney System. Am J Surg Patbol 1996; 20: 1161-81.

33. Felten A, Barreau C, Bizet C, Lagrange PH, Philippon A. Lactobacillus species identification, $\mathrm{H}_{2} \mathrm{O}_{2}$ production, and antibiotic resistance and correlation with human clinical status. J Clin Microbiol 1999; 37: 729-33.

34. Pérez P, Minnaard Y, Disalvo E, De Antoni G. Surface Properties of Bifidobacterial Strains of Human Origin. Appl Environ Microbiol 1998; 64: 21-6.

35. Figueroa G, Acuña R, Troncoso M, Portell DP, Toledo MS, Valenzuela J. Helicobacter pylori infection in Chile. Clin Infect Dis 1997; 25: 983-9.

36. PRAdo V. Enfermedades infecciosas emergentes: ¿Un problema nuevo? Rev Méd Chile 1996; 124: 7-10.

37. Roberts P, Dickinson R, Whitehead A, Laughton C, FOWERAKER J. The culture of lactobacilli species in gastric carcinoma. J Clin Pathol 2002; 55: 477.

38. Gotteland M, Cruchet S. Suppressive effect of frequent ingestión of Lactobacillus jobnsonii La1 on Helicobacter pylori colonization in asymptomatic volunteers. J Antimicrob Chemother 2003; 51: 1317-19.

39. SaKamoto I, Igarashi M, Kimura K, Takagi A, Miwa T, Koga Y. Suppresive effects of Lactobacillus gasseri OLL 2716 (LG21) on Helicobacter pylori infection in humans. J Antimicrob Chemother 2001; 47: 709-10.

40. SU YC, WANG WM, WANG KY. Reply to M Gotteland et al. Am J Clin Nutr 2005; 81: 939-40. 